\title{
WHO's Chan warns over spread of Zika virus beyond Latin America
}

\author{
Anne Gulland
}

London

The world will face a "severe public health crisis" if the Zika virus spreads beyond Latin America, the World Health Organization's director general has said.

Margaret Chan told a press conference that no one can currently predict whether the virus and its association with fetal malformations and neurological complications, as seen in Brazil and other parts of Latin America, will spread to other parts of the world.

She added that a "shift in our thinking is needed, away from the management of individual cases and towards the longer term building of capacities in countries to cope with these added burdens. Fetal malformations place a heartbreaking strain on families and communities, as well as systems for healthcare and social support."

WHO said that Zika virus transmission has been reported in 33 countries and territories of the Americas and that Cuba and Dominica were the latest to report local transmission. An increase in the number of cases of microcephaly has been reported in Brazil and French Polynesia, and an increased incidence of Guillain-Barré syndrome has been reported in 12 countries.

News reports have stated that Panama reported its first case of microcephaly linked to the Zika virus on 19 March, although this has not yet been confirmed by WHO.

Christopher Dye, director of strategy, policy, and information at WHO, told the press conference that studies currently suggest that about $1 \%$ of cases of the infection will lead to severe neurological disorders.

"We know already that there are thousands of cases of microcephaly in just one part of Brazil, so the expectation across the Americas as a whole is of many more thousands of cases, and that is going to present a very large burden of illness which is going to require a substantial investment," he said.

Anthony Costello, director of the maternal, newborn, child and adolescent health department at WHO, said that suspected cases of microcephaly in Brazil had been confirmed by CT (computed tomography) scans. Of 6480 suspected cases, most of them in the north east, 2212 have been investigated and 863 confirmed. The conversion rate from suspected to confirmed cases is $39 \%$. "If that rate continues we would expect that just over 2500 cases will emerge of babies with brain damage and with clinical signs of microcephaly," said Costello. He added that a changing pattern of birth defects had been noted, including hearing and visual defects.

Chan said that it was important to be cautious about projecting numbers of cases. "Estimates of the rates will become much better over time," she added.

Experts agree that a reliable, point of care diagnostic test is an urgent requirement, said Chan. At present around 30 companies are working on or have developed such a test.

Some 23 groups in Austria, Brazil, France, India, and the United States are looking at developing a vaccine that is safe enough to be used in pregnant women and women of childbearing age. However, Chan acknowledged that the first clinical trials will begin only by the end of this year, by which time this "explosive wave" of the spread of the virus may be over.

"All scientists agree that the development of a vaccine is imperative, as more than half of the world's population lives in an area where the Aedes aegypti mosquito [the vector for Zika] is present," she said. 